\title{
Congenital disorders of glycosylation
}

\author{
Irene J. Chang ${ }^{1}$, Miao $\mathrm{He}^{2}$, Christina T. Lam $^{1}$ \\ ${ }^{1}$ Division of Biochemical Genetics, Department of Pediatrics, University of Washington, Seattle, Washington, USA; ${ }^{2}$ Department of Pathology and \\ Laboratory Medicine, The Children's Hospital of Philadelphia, Philadelphia, Pennsylvania, USA \\ Contributions: (I) Conception and design: All authors; (II) Administrative support: None; (III) Provision of study materials or patients: None; (IV) \\ Collection and assembly of data: None; (V) Data analysis and interpretation: None; (VI) Manuscript writing: All authors; (VII) Final approval of \\ manuscript: All authors. \\ Correspondence to: Irene J. Chang, MD. Division of Biochemical Genetics, Department of Pediatrics, University of Washington, Seattle, Washington, \\ USA. Email: ijchang@uw.edu.
}

\begin{abstract}
Congenital disorders of glycosylation are a genetically and clinically heterogeneous group of $>130$ diseases caused by defects in various steps along glycan modification pathways. The vast majority of these monogenic diseases are autosomal recessive and have multi-systemic manifestations, mainly growth failure, developmental delay, facial dysmorphisms, and variable coagulation and endocrine abnormalities. Carbohydrate deficient transferrin (CDT) and protein-linked glycan analysis with mass spectrometry can diagnose some subtypes of congenital disorders of glycosylation (CDG), while many currently rely on massively parallel genomic sequencing for diagnosis. Early detection is important, as a few of these disorders are treatable. Molecular and biochemical techniques continue to further our understanding of this rapidly expanding group of clinically and genetically diverse disorders.
\end{abstract}

Keywords: Congenital disorders of glycosylation; carbohydrate deficient transferrin; carbohydrate deficient glycoprotein syndrome; PMM2-CDG; MPI-CDG; ALG6-CDG; N-linked glycosylation; O-linked glycosylation; lipid glycosylation defects; congenital disorders of glycosylation (CDG)

Submitted Aug 12, 2018. Accepted for publication Oct 17, 2018.

doi: $10.21037 /$ atm.2018.10.45

View this article at: http://dx.doi.org/10.21037/atm.2018.10.45

\section{Overview}

Glycosylation is the process of adding sugar residues to proteins and lipids in different cellular pathways. Congenital disorders of glycosylation (CDG) are a genetically and clinically heterogeneous group of over a hundred diseases caused by defects in various steps along glycan synthesis or modification pathways. Most of these monogenic diseases are autosomal recessive in inheritance, but autosomal dominant and $\mathrm{X}$-linked forms have also been described.

CDG typically present with multi-systemic manifestations, most commonly developmental delay, failure to thrive, hypotonia, neurologic abnormalities, hepatopathy, and coagulopathy. Affected individuals may also present with eye, skin, and cardiac disease, as well as facial dysmorphisms. Although neurologic changes and cognitive delays are seen in the majority of affected individuals, there are certain cases and even types that do not have neurologic manifestations. Given the broad clinical and genetic etiology of CDG, clinical diagnosis relies on high index of suspicion in multi-systemic disease.

Serum carbohydrate deficient transferrin (CDT) analysis is the first-line screening test in patients with suspected CDG, but is limited in detection to $\mathrm{N}$-glycosylation defects with sialic acid deficiencies. Next-line testing includes dolichol-linked glycan analysis and genetic testing. Early diagnosis of this group of exponentially growing diseases is important, as some CDGs are treatable. Treatment for glycosylation defects is mainly supportive, although targeted therapies are available for MPI-CDG, SLC35C1CDG, PIGM-CDG, and PGM1-CDG. Details about these treatments are under the "Targeted Therapies and Prognosis" section below. The focus of this review will be on the most common types of CDG with recognizable 
phenotypes or treatments, with the target audience being primary care providers.

\section{Epidemiology}

The incidence and prevalence of all types of CDG in aggregate have not been well established, although patients have been reported worldwide from almost every ethnic background and both sexes are equally affected. The estimated prevalence in European and African American populations is 1/10,000 based on carrier frequencies of known pathogenic variants in 53 genes (1-4). The prevalence of the most commonly diagnosed CDG, PMM2-CDG, ranges from 1/20,000 in Dutch populations and 1/77,000 in Estonia based on isolated reports $(5,6)$. To date, fewer than 100 cases have been reported for most CDG types.

\section{Biochemical classification and nomenclature}

Broadly, CDG currently are classified into four categories(I) N-linked glycosylation, (II) O-linked glycosylation, (III) combined $\mathrm{N}$ - and O-linked/multiple glycosylation, and (IV) lipid and glycosylphosphatidylinositol (GPI) anchor biosynthesis defects.

The N-linked protein glycosylation defect PMM2-CDG, previously known as CDG type Ia, was the first CDG to be reported by Jaeken in 1980 and remains by far the most common CDG to date (7). PMM2-CDG was initially named "carbohydrate-deficient glycoprotein syndrome" due to multiple serum glycoprotein abnormalities seen by isoelectric focusing of serum transferrin in affected individuals. Historically, CDG were classified by patterns of transferrin isoform analysis-type I patterns were attributed to dolichol-linked glycan assembly and transfer defects localizing to the cytoplasm or ER, and type II patterns were attributed to processing defects in the Golgi apparatus. From this branch point, CDGs were then named alphabetically in order of discovery.

With the advent of widespread molecular diagnostics, CDG nomenclature was updated in 2008 to specify molecular etiology of disease, reflecting the exponential growth of pathways and disorders that did not neatly fit in the previously established dichotomous categories. Presently, CDG nomenclature is denoted by the affected gene name (non-italicized, gene names at www.genenames. org), followed by -CDG (e.g., PMM2-CDG) (8).

\section{Genetics}

The vast majority of congenital disorders of glycosylation are inherited in an autosomal recessive fashion, with one mutation inherited from each asymptomatic (carrier) parent. Molecular testing, usually with next-generation sequencing methods, is necessary to establish a genetic diagnosis. Parental testing for the known variant can confirm inheritance versus de novo occurrence. For autosomal recessive inheritance, the recurrence risk for siblings and each pregnancy of an affected individual is $25 \%$ for being affected, $50 \%$ for being an asymptomatic carrier, and $25 \%$ for being unaffected.

A handful of CDG have autosomal dominant inheritance (N-linked: GANAB-CDG, PRKCSH-CDG; O-linked: EXT1/EXT2-CDG, POFUT1-CDG, POGLUT1-CDG). Fewer are X-linked (ALG13-CDG, SSR4-CDG, PIGACDG, SLC35A2-CDG, ATP6AP1-CDG). Most dominant and some $\mathrm{X}$-linked forms of CDG are due to de novo mutations. Specific diseases and genes are described below in the "pathophysiology" section.

Mutation data for all published genes for CDG are available at the Human Gene Mutation Database (http:// www.hgmd.cf.ac.uk/ac/index.php). Information about specific gene variants is available at the Leiden Open Variation Database with integrated in silico pathogenicity tools (http://www.lovd.nl/3.0/home). Clinical synopses for specific genes can be found at the Online Mendelian Inheritance in Man (http://www.omim.org/) or in a more limited scope at GeneReviews (http://www.ncbi.nlm.nih. gov/books/NBK1116/). Given the small number of affected patients for most CDG subtypes, genotype-phenotype correlation is difficult to establish.

\section{Pathophysiology}

Over 130 types of CDG have been reported to date $(9,10)$. Given the ubiquitous presence of glycosylation pathways, CDG are extremely diverse in their biochemical pathogenesis. Numerous proteins and lipids (i.e., sphingolipids and glycolipids) undergo glycosylation with monosaccharides and/or oligosaccharides, collectively termed glycans, in different cellular compartments. Their subcellular locations are diverse, but most defects occur within the ER or Golgi apparatus. Clinical features and genetic etiology of more common CDG by pathway is summarized in Table S1. 
Amongst proteins, glycans are described by their linkage to the polypeptide chain- $\mathrm{N}$-glycans are attached to the amide group of asparagine (Asn) while O-glycans are attached to the hydroxyl group of either serine or threonine. $\mathrm{N}$-glycan synthesis requires the stepwise construction of nucleotide-linked sugars in the cytosol, assembly in the endoplasmic reticulum, and processing in the Golgi apparatus. In contrast, O-glycan synthesis requires assembly but no processing, therefore O-glycosylation defects occur predominantly in the Golgi apparatus.

\section{N-linked protein glycosylation defects}

$\mathrm{N}$-glycosylation involves the covalent attachment of carbohydrate structures to the side chain amide group of Asn residues within a consensus Asn-X-Ser/Thr acceptor site, translocation of the substrate polypeptide to the endoplasmic reticulum for remodeling, and further modification of the $\mathrm{N}$-glycan chain within the Golgi $(11,12)$. Defects anywhere along the synthesis, assembly, and processing pathway can lead to clinical disease.

PMM2-CDG is caused by pathogenic variants in the phosphomannomutase 2 (PMM2) gene, leading to deficiency of the PMM2 enzyme that catalyzes the cytosolic conversion of mannose-6-phosphate to mannose-1-phosphate in the second step of guanosine diphosphate (GDP) mannose synthesis. Most patients harbor compound heterozygous pathogenic missense mutations (www.lovd.nl/PMM2). The most common recurrent pathogenic variant p.Arg141His is found in approximately $40 \%$ of affected individuals of European ancestry, and p.Phe119Leu is also frequently found in northern Europe (1). Genotype-phenotype correlations have been reported for PMM2-CDG $(3,13,14)$.

MPI-CDG is an autosomal recessive disorder caused by pathogenic variants in the mannose phosphate isomerase (MPI) gene leading to deficient phosphomannose isomerase (MPI). MPI normally catalyzes the first step of GDPmannose synthesis (i.e., the conversion of fructose-6phosphate to mannose-6-phosphate), but fructose-6phosphate does not accumulate intracellularly since it can also be metabolized by the glycolytic pathway. Therefore, although biochemically similar to PMM2-CDG, MPICDG does not cause as significant neurologic and multisystemic involvement. CDT is also the screening test of choice for MPI-CDG, which shows a type 1 pattern. The diagnosis can then be confirmed molecularly or by fibroblast/leukocyte MPI activity.
ALG6-CDG is a recessive disease caused by mutations in $A L G 6$, leading to abnormal attachment of three glucose molecules to dolichol-linked mannose intermediates and downstream hypoglycosylation of serum glycoproteins (15).

\section{O-linked glycosylation defects and combined $\mathbf{N}$ - and $\mathbf{O}$-linked glycosylation defects}

O-glycosylation comprises the stepwise addition of carbohydrate chains to serine, threonine, and hydroxylysine residues of proteins by glycosyltransferases in the Golgi apparatus (16). Several types of O-linked glycans have been associated with human disease, named by the first sugar attached to the amino acid residue [e.g., O-Nacetylglucosaminyl for GlcNAc and O-N-acetylgalactosaminyl for $\mathrm{N}$-acetylgalactosamine (GalNAc)] (17).

\section{Lipid glycosylation and GPI anchor biosynthesis defects}

GPI anchors are glycolipids that undergo sequential assembly in the endoplasmic reticulum and modifications within the Golgi. GPI anchor biosynthesis disorders due to enzyme deficiencies are named alphabetically by order of discovery and not chronologically by step of assembly. Once synthesized, GPI anchors reside on plasma membranes and bind hundreds of cell-surface proteins, performing a plethora of cellular functions. Most of these diseases are autosomal recessive with the notable exception of $\mathrm{X}$-linked PIGA deficiency.

\section{Clinical manifestations}

Given the ubiquitous presence of glycosylation pathways, virtually any organ system may be involved in CDG, although most cases involve neurologic abnormalities. Some CDG present with ichthyosis, including MPDU1-CDG, DOLK-CDG, SRD5A3-CDG (Figure 1), and PIGLCDG $(18,19)$. Almost all CDG present with multi-systemic disease within the first few years of life, except some affect only a single organ system (i.e., retina in DHDDS-CDG; neuromuscular junction in ALG2-CDG, ALG14-CDG, CFPT1-CDG; brain in ST3GAL3-CDG, TUSC3-CDG; skin or skeletal muscle in POGLUT1-CDG, POFUT1CDG; cartilage in EXT1/EXT2-CDG; liver in TMEM199CDG; red blood cells in SEC23B-CDG). The age of onset and severity may range from neonatal lethal to nearly asymptomatic adulthood, and any permutation in between. 

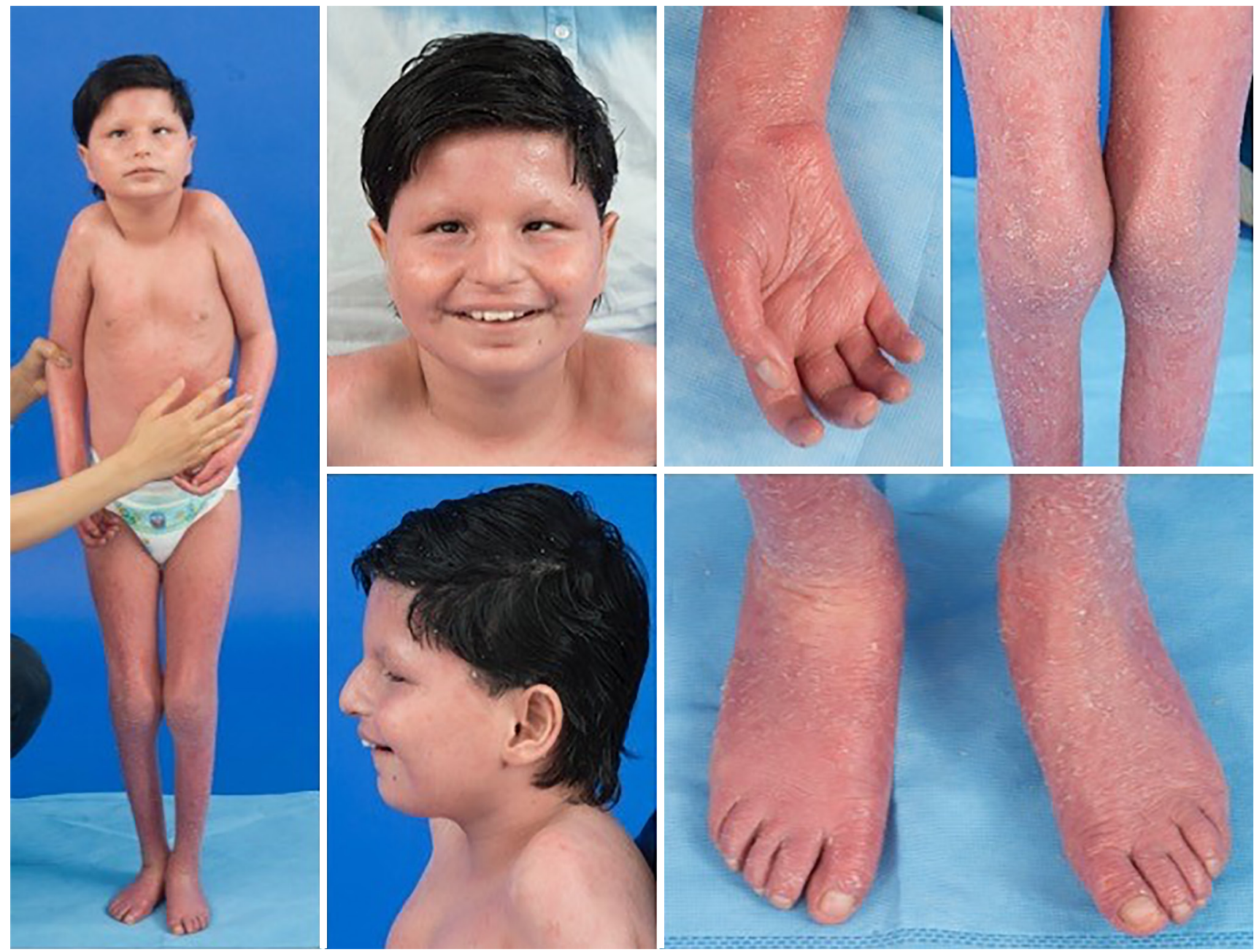

Figure 1 Ichthyosis, hypotonia, and strabismus in a patient with SRD5A3-CDG. Courtesy of NIH, Lynne Wolfe, CRNP, Donna Krasnewich, MD, PhD.

The most commonly reported constellation of symptoms includes developmental delay, failure to thrive, hypotonia, neurologic abnormalities, hypoglycemia, and variable liver, eye, skin, gastrointestinal, immunologic, skeletal, and coagulation abnormalities (19).

The complete phenotype for many CDG subtypes remains to be fully delineated due to the rarity of reported cases. Therefore, CDG should be considered in any setting of multi-systemic disease, especially in cases with a neurologic component or nonspecific developmental delay with unclear etiology.

Although the pathophysiology of multitude symptoms remains to be elucidated, the relationship between certain glycosylation pathways and specific clinical symptoms has been clarified. For instance, failure to thrive seen in many types of CDG is attributable to the hypoglycosylation and impaired formation of several glycoproteins within the insulin growth pathway including IGF-1, ALS, and IGFBP-3 (20). As we better understand this group of complex disorders, CDG are increasingly recognized in individuals with elusive diagnoses. The organ system involvements of different CDG are summarized in Table S1. We will discuss the clinical features of most common forms and forms with targeted treatments of CDG below.

\section{N-linked protein glycosylation defects}

As the most commonly diagnosed CDG, the phenotype of 

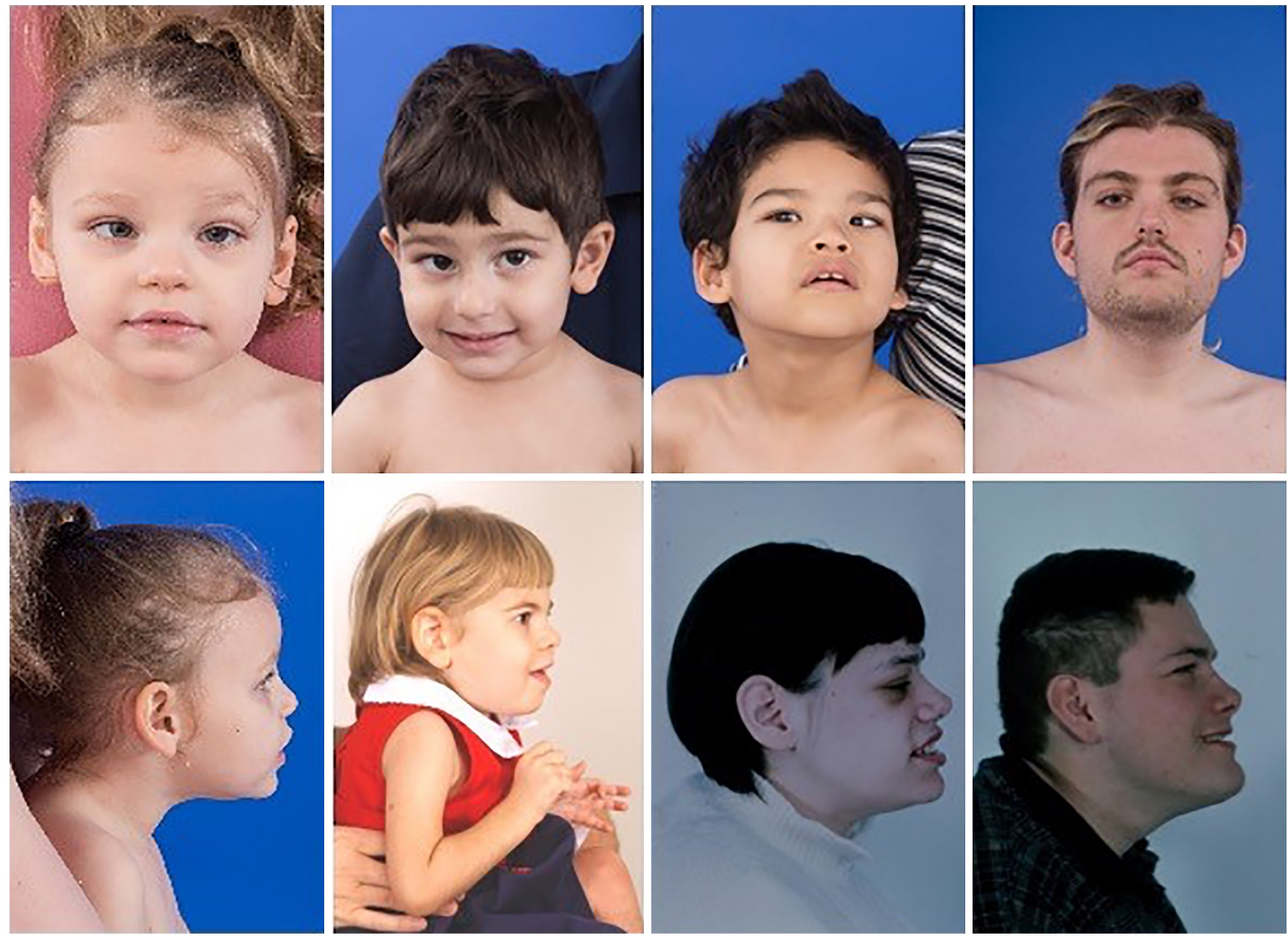

Figure 2 Clinical portraits of patients with PMM2-CDG from childhood to adulthood (left to right). Esotropia, long philtrum, large, hypoplastic or dysplastic ears, hypotonic facies, and downslanting palpebral fissures are seen (top row). Profile view shows the prominent chin, which is more pronounced with age (bottom row). Courtesy of NIH, Lynne Wolfe, CRNP, Donna Krasnewich, MD, PhD.

N-linked glycosylation disorders is often heralded as the classic presentation. However, the phenotypic spectrum of CDG is quite diverse, and many CDG may not present with stereotypical symptoms associated with PMM2-CDG.

\section{PMM2-CDG (CDG-Ia, PMM2 deficiency)}

PMM2-CDG is the most common CDG, with over 700 reported cases worldwide. It is characterized by multisystemic severe disease in infancy, neurologic disease and developmental delay in childhood, and/or stable intellectual disability in adulthood $(21,22)$.

In infancy, PMM2-CDG presents with neurologic abnormalities typically shortly after birth, namely strabismus and abnormal eye movements, cerebellar hypoplasia, hypotonia, psychomotor retardation, ataxia, hypotonia, and hyporeflexia. Infants may also have hepatic disease, nephrotic syndrome and renal cysts, pericardial effusion and hypertrophic cardiomyopathy, failure to thrive, and multi-organ failure resulting in death within the first year of life in up to $20 \%$ of affected individuals (21,23-28).

A constellation of dysmorphic features has been described in patients with PMM2-CDG (Figures 2,3). These include a hypoplastic cerebellum, facial dysmorphisms (i.e., large, dysplastic ears), inverted nipples, and abnormal distribution of adipose tissue over the buttocks or suprapubic region which may resolve with age $(14,21,29-32)$. Patients have been described to have an outgoing and happy demeanor. 

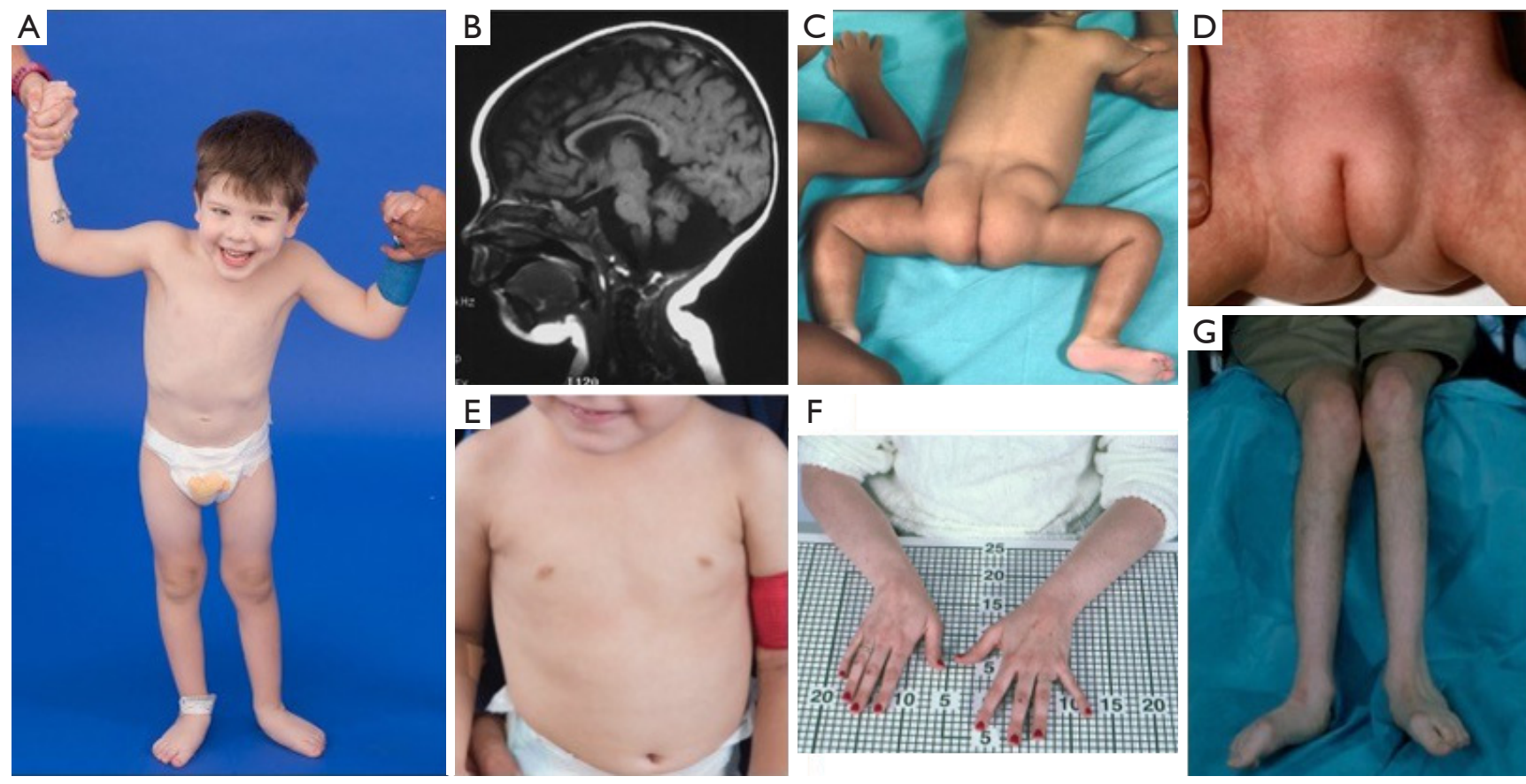

Figure 3 Clinical photographs of patients with PMM2-CDG. (A) Happy demeanor. Truncal hypotonia and kyphoscoliosis are often present; (B) MRI showing cerebellar vermis hypoplasia; (C) posterior suprapelvic fat pads present in approximately $25 \%$ of patients with PMM2CDG; (D) suprapubic fat pad seen in infancy, usually disappears by 5 years of age; (E) inverted nipples; (F,G) long thin fingers and atrophied low extremities secondary to demyelinating peripheral neuropathy. Courtesy of NIH, Lynne Wolfe, CRNP, Donna Krasnewich, MD, PhD. MRI, magnetic resonance imaging.

Presentation is highly variable, although strabismus can be seen in more than $70 \%$ of affected patients $(21,23,33-35)$. Inverted nipples and abnormal fat pads are seen in about $25-50 \%$ of patients (36).

In childhood, affected individuals may develop retinitis pigmentosa, stroke-like episodes and seizures, speech and motor delays, and peripheral neuropathy. Constitutionally, patients commonly have failure to thrive due to feeding and GI abnormalities and global developmental delay. Elevated liver transaminases without clinical consequence may be observed, which typically normalize by 5 years of age with occasional fluctuations with illness $(21,24)$. Liver biopsies are rarely indicated in CDG unless hepatic fibrosis is suspected (1). Clinical hypothyroidism is rare, but patients with CDG should have their thyroid hormones and free T4 measured, which may show low thyroid binding globulin (TBG) and transient elevations in thyroid stimulating hormone (TSH) (37). Liver and bile duct malformations have not been reported in PMM2-CDG patients.

Adults with PMM2-CDG may live until their $7^{\text {th }}$ or $8^{\text {th }}$ decades with stable cognitive delay, peripheral neuropathy, and progressive thoracic and spinal kyphoscoliosis with osteopenia or osteoporosis (34). Cerebellar ataxia is an increasingly recognized symptom along with multisystemic involvement (38-40). Endocrine abnormalities including hyperprolactinemia, growth hormone release with hyperglycemia, insulin resistance, and hyperinsulinemic hypoglycemia $(41,42)$. In affected women, hypogonadotropic hypogonadism may lead to absent secondary sexual development or absent ovaries $(41,43,44)$. Patients may be at increased risk of thrombosis due to decreased serum coagulation factors including factors IV, IX, and XI, antithrombin III, protein C, and protein S (29).

\section{MPI-CDG (CDG-Ib, mannosephosphate isomerase deficiency)}

MPI-CDG is unique because affected patients have little to no neurologic involvement and some manifestations of the disease are treatable by oral mannose (2). Symptoms are mainly hepatic-intestinal without dysmorphic features or cognitive delays. Patients typically present with recurrent 
vomiting, significant hypoglycemia, failure to thrive, potentially life-threatening protein-wasting enteropathy, liver fibrotic changes, and bile duct dilatation (45-51). Patients are at increased risk for thrombotic events due to low serum concentrations of protein $\mathrm{C}$ and $\mathrm{S}$, and antithrombin III.

\section{ALG6-CDG (glucosyltransferase 1 deficiency)}

ALG6-CDG is the second most common N-glycosylation defect characterized by similar but milder phenotype than PMM2-CDG. Patients with ALG6-CDG have failure to thrive, developmental delay, hypotonia, seizures, strabismus, ataxia, coagulopathy, and facial dysmorphisms (i.e., low-set ears, hypertelorism, and macroglossia). Similar to MPI-CDG, they may also have protein-losing enteropathy. Additionally, affected patients may have skeletal abnormalities including brachydactyly and finger malformations and scoliosis. Affected patients typically do not typically have retinitis pigmentosa or cerebellar hypoplasia (52).

\section{O-linked glycosylation defects and combined $\mathbf{N}$ - and O-linked glycosylation defects}

Due to the substantial presence of O-glycans in mucincontaining proteins including glycosaminoglycans (GAGs) and epithelial surfaces (53), disorders of GAG synthesis typically lead to skeletal dysplasias or connective tissue disease. Affected patients may present with musculoskeletal, skin, and joint abnormalities (e.g., joint laxity, multiple exostoses, chondro/osteosarcomas) in addition to neurologic symptoms (54-56). For example, $\mathrm{N}$-acetylga lactosaminyltransferase 3 (GALNT3) O-glycosylates the phosphaturic hormone, FGF23, preventing proteolytic cleavage and allowing its intact secretion. GALNT3deficiency leads to familial tumoral calcinosis, characterized by hyperphosphatemia and ectopic calcifications $(57,58)$.

\section{Lipid glycosylation and GPI anchor biosynthesis defects}

Glycosphingolipids and their sialylated derivatives, gangliosides, are mainly expressed by neurons. Defects in the breakdown of gangliosides lead to accumulation and the well-characterized lysosomal storage diseases. On the opposite end, defects in ganglioside biosynthesis such as ST3GAL5-CDG and B4GALNT1-CDG are exceedingly rare and lead to severe neurodegenerative disease. Patients may present with spastic paraplegia, severe intellectual delay, epilepsy, and non-neurologic symptoms including skeletal dysplasia, dysmorphic features, and abnormal skin pigmentation $(59,60)$.

Mutations in many genes within the GPI anchor biosynthetic pathway cause a variety of multiple congenital anomalies, intellectual disability, and epilepsy. The best-characterized GPI biosynthesis defect, X-linked PIGA deficiency, presents with infantile spasms with hypsarrhythmia, hypotonia, multiple brain abnormalities, and facial dysmorphisms. Patients may also have variable skin, liver, heart, and renal disease (61-69). Some mutations within PIGA cause the phenotypically distinct disease paroxysmal nocturnal hemoglobinuria $(\mathrm{PNH})$, an acquired disorder of bone marrow failure $(70,71)$.

\section{Diagnosis}

When a CDG is clinically suspected, the first step is to order biochemical CDG testing in plasma or serum, including CDT and $\mathrm{N}$-glycan testing. Serum CDT and $\mathrm{N}$-glycan analysis can only detect $\mathrm{N}$-glycosylation defects, therefore they would not be useful in differentiating isolated O-glycosylation or GPI anchor defects. Transferrin isoform analysis was originally obtained by isoelectric focusing of transferrin, as failure of $\mathrm{N}$-glycan synthesis causes partial deficiency of sialic acid, which alters the charge on serum transferrin and subsequently its cathodal migration on an electrophoretic field. However, mass spectrometry-based analysis of transferrin and $\mathrm{N}$-glycan have now largely replaced isoelectric focusing by identifying specific changes in oligosaccharides by mass and charge (72).

\section{$\mathbf{N}$-linked protein glycosylation defects}

Serum transferrin CDT results are reported as the ratio of mono-oligosaccharide/di-oligosaccharide transferrin, a-oligosaccharide/di-oligosaccharide transferrin, trisialo/di-oligosaccharide transferrin, apolipoprotein CIII1/apolipoprotein CIII-2, and apolipoprotein CIII-0/ apolipoprotein CIII-2 ratio. These quantitative results will also come with an interpretation of the pattern of findings.

A type I pattern transferrin CDT is characterized by increased di- and asialotransferrin bands, and indicates defects in $\mathrm{N}$-glycan synthesis in the cytosol or endoplasmic reticulum. A type II pattern is characterized by increased di- and asialotransferrin bands, and tri- and/ 
or monosialotransferrin bands, and indicates defects in $\mathrm{N}$-glycan processing in the Golgi apparatus (73).

If a type I serum transferrin CDT pattern is detected, PMM2 deficiency or MPI deficiency should be at the forefront of differentials, as PMM2-CDG is the most common CDG and MPI-CDG is treatable and potentially fatal if left untreated. To differentiate between diagnoses, $\mathrm{N}$-glycan profiling, molecular sequencing, or enzymatic testing should be undertaken. The diagnosis of PMM2CDG or MPI-CDG is confirmed through molecular testing showing biallelic pathogenic variants in PMM2 or MPI, followed by PMM or MPI enzyme activity in leukocytes or fibroblasts if the pathogenicity of genetic variants are uncertain. N-glycan analysis or molecular analysis would differentiate the majority of ALG-CDG from PMM2 or MPI-CDG (15).

A type II serum transferrin CDT pattern indicates Golgi defects such as N-acetylglucosaminyltransferase (GnT) II deficiency (CDG type IIA, MGAT2-CDG). Apolipoprotein CIII (Apo-CIII) isoform analysis is a complementary test for a type II CDT profile, as it measures mucin type O-glycosylation defects in the Golgi apparatus. There is limited sensitivity for CDT or Apo-CIII in detecting type II CDG. Thus N-glycan and O-glycan profiling and molecular panel or exome sequencing should be undertaken when these clinical tests are available. Transferrin glycosylation patterns may normalize sporadically; therefore, repeated testing may be indicated in patients with high index of suspicion. False positives may be obtained in patients with acute crisis of hereditary fructose intolerance, galactosemia, acute liver disease, and some bacterial infections. None of the biochemical CDG tests can screen for all CDGs, thus even in the presence of normal screening results, molecular gene panel testing or exome sequencing may be performed for strong clinical suspicion. Conversely, biochemical and functional confirmation of molecular genetic findings are also essential as the majority of patients with CDG carry at least one mild and often novel missense mutation.

\section{O-linked glycosylation defects and combined $\mathbf{N}$ - and O-linked glycosylation defects}

Diagnosis relies on molecular sequencing, as transferrin isoform analysis would not detect isolated $\mathrm{O}$-glycosylation defects. Combined N- and O-linked glycosylation defects can be detected by CDT, ApoCIII analysis, and plasma $\mathrm{N}$-glycan and $\mathrm{O}$-glycan analysis.

\section{Lipid glycosylation and GPI anchor biosynthesis defects}

Flow cytometry of blood granulocytes measures cell surface expression of GPI-anchored proteins such as CD16 and CD24. Flow cytometry analysis of white blood cells or red blood cells for certain GPI-anchored cell surface proteins are available clinically as a test for $\mathrm{PNH}$ due to acquired mutations in the PIGA gene. The PNH test may reveal abnormalities in other GPI anchor deficiencies, but diagnosis mostly relies on molecular analysis.

\section{Molecular analysis}

The highest diagnostic yield for CDG is next-generation based gene sequencing panel or clinical exome sequencing (CES). Genetic sequencing proofreads the nucleotide sequence, or "letter" spelling, of genes to determine whether there is a change that affects the function of the gene. The human genome consists of 3 million nucleotides but only $1-2 \%$ of these, called exons, are translated into a functional protein product. The remaining noncoding DNA interspersed between exons that do not get translated are called introns (74). CES examines almost all known exons of the approximately 20,000 genes in the human genome, which accounts for a minority of the genetic material in chromosomes but are most likely to contain disease-causing (pathogenic) variants. CES can also include mitochondrial DNA (mtDNA) sequencing, which interrogates small, extranuclear, circular DNA located in the mitochondria that is exclusively maternally-inherited.

The possible results for CES include positive, negative, and variants of unknown significance. A positive result means known disease-causing (i.e., pathogenic) variants are identified, after which the diagnosis, natural history, prognosis, recurrence risk, and treatment options can be discussed. A negative result means no detectable pathogenic variants were identified. Variants of unknown significance (a.k.a., VUS) means that although genetic changes were identified, there is not enough information about the specific genetic change to know definitively if it is diseasecausing. Variations in each individual's DNA are expected, thus having parental samples tested concurrently for comparison can assist laboratory and clinical interpretation of results. The diagnostic yield of CES is estimated at $30-35 \%$ and is increasing over time as gene discovery and knowledge about the human genome continue to progress (75-77). CES is increasingly ordered as the first-line broad 
genetic testing of choice given its quick turnaround time and low relative cost for amount of genetic information analyzed. The limitations of CES include the lack of $100 \%$ sensitivity, the inability to detect certain types of genetic changes (e.g., deletions, duplications, trinucleotide repeats, deep intronic mutations, or methylation defects), and the fact that a diagnosis may not provide additional information about the disease or change management.

In the reporting of CES, incidentally-detected pathogenic variants in genes associated with well-known genetic conditions may be reported as secondary findings (78). This list of recommended diseases is curated by the American College of Medical Genetics (ACMG). The Genetic Information Nondiscrimination Act (GINA) is an important consideration when deciding whether to opt in or out of learning incidental findings (79). GINA protects individuals from the misuse of genetic information in health insurance and employment, but not life insurance. GINA protects the following genetic information: family medical history, carrier testing, prenatal genetic testing, susceptibility and predictive testing, and analysis of tumors or other assessments of gene, mutations, or chromosomal changes.

\section{Management}

The management of CDG depends largely upon the individual's specific symptoms. Recurrent symptoms in patients with CDG include failure to thrive, global developmental delay, vomiting, stroke-like episodes, and skeletal abnormalities. Clinical or sub-clinical coagulopathy, endocrinopathy, hepatopathy, and cardiac defects are also commonly seen. Baseline laboratory tests to establish the extent of disease and routine monitoring are recommended, especially for PMM2-CDG. These include liver function tests, serum albumin, thyroid function tests including free $\mathrm{T} 4$, protein $\mathrm{C}$, protein S, antithrombin III, factor IX, urinalysis, and serum gonadotropins and growth hormone.

Recommended imaging includes echocardiogram, renal ultrasound, bone age, ophthalmologic examination for evaluation of lens, retina, ocular mobility, and intraocular pressure. Unless otherwise indicated, routine vaccinations are recommended for adults and children affected with CDG. Antibody titers should be obtained after vaccination as patients may have a suboptimal immunogenic response. Prophylactic repletion of coagulation factors prior to any surgical procedures may be necessary if deficiencies exist at baseline.

Clinical genetics evaluation should be undertaken to discuss the hereditary aspects of CDG, as well as establishing a medical home for these complex patients. The medical home is typically the biochemical genetics service, although genetics, neurodevelopment, or neurology departments have also served in this capacity if a dedicated biochemical genetics service is unavailable. Specialist referral for gastroenterology, hematology, endocrinology, nutrition support, speech, occupational, physical, and feeding therapies, orthopedics, and rehabilitation medicine are often necessary.

\section{Targeted therapies and prognosis}

Treatment for the majority of CDG types is largely supportive, with a few exceptions. MPI-CDG is the most effectively treatable of all CDG. Oral mannose is converted to mannose-6-phosphate by intracellular hexokinases, therefore bypassing the enzymatic block and producing the deficient substrate. Mannose supplementation typically starts at $1 \mathrm{~g} / \mathrm{kg}$ body weight per day, divided into 4-6 doses a day. While the potentially life-threatening protein-wasting enteropathy is especially responsive to mannose treatment, the liver disease in MPI-CDG may continue to progress. Clinical symptoms improve rapidly and transferrin CDT normalize over months, although liver disease may continue to progress with treatment $(45,80,81)$.

Caution should be exercised in supplementing mannose during pregnancy, as mannose administration in pregnant hypomorphic phosphomannose isomerase mouse models resulted in embryonic lethality and blindness in their pups (82). In addition, intravenous mannose has been associated with decreased consciousness and seizures, which resolved with glucose administration (83).

The treatment for PMM2-CDG is largely supportive and based on symptomatology. However, upcoming clinical trials on mannose-1-phosphate substrate replacement therapy are currently under development.

For other CDG, various oral simple sugars have been investigated with the aim of theoretically improving hypoglycosylation. Fucose has been tried for SLC35C1CDG and galactose for PGM1-CDG and SLC35A2-CDG with mixed results (84). D-galactose at $1.0-2.5 \mathrm{~g} / \mathrm{kg} / \mathrm{day}$ ( $\max 50$ grams) has been demonstrated to improve the hypoglycemia, coagulopathy, and endocrinopathy in PGM1CDG $(85,86)$. Galactose has also been shown to improve the endocrinopathy and coagulopathy in TMEM165CDG (87) and SLC39A8-CDG. Considerable clinical improvement was also reported in SLC39A8-CDG patients 
on $15-20 \mathrm{mg} / \mathrm{kg} /$ day of $\mathrm{MnSO}_{4}$ (88). Clinical trials are ongoing to investigate the utility of $\mathrm{N}$-acetylmannosamine (ManNAc) in GNE-CDG (89), and several pre-clinical trials are underway for other CDG (90).

Despite medical strides, significant mortality exists for children with CDG within the first year of life from multiorgan failure or severe infection (91). Infants with CDG may present with fulminant multi-organ disease, intractable seizures, or severe hypoalbuminemia progressing to anasarca. Some patients respond to aggressive diuresis and albumin replacement while others are refractive to treatment. Sodium butyrate has been demonstrated to improve seizure control in CAD-CDG and PIGM-CDG (92). Ketogenic diet has also been shown to decrease seizure frequency in some cases of PIGA-CDG (93). During stroke-like episodes, intravenous hydration and maintenance of normal blood glucose may be helpful while underlying vascular thrombotic or bleeding etiology is ruled out.

With the advent of genome-editing techniques and better understanding of the mechanism of diseases encompassed by the diagnostic umbrella of CDG, the future of targeted therapeutic development remains promising.

\section{Acknowledgements}

We would like to thank Lynne Wolfe, ARNP and Donna Krasnewich, MD, $\mathrm{PhD}$ for providing us with clinical photos obtained as part of the Clinical and Basic Investigations Into Known and Suspected Congenital Disorders of Glycosylation (NCT02089789). We would also like to thank Jenny Thies, MS, LGC for her expertise in genetic counseling.

Funding: IJ Chang is supported by the National Institutes of Health T32GM007454.

\section{Footnote}

Conflicts of Interest: The authors have no conflicts of interest to declare.

Informed Consent: Written informed consent was obtained from the patients for publication of this manuscript and any accompanying images.

\section{References}

1. Jaeken J, Matthijs G. Congenital disorders of glycosylation. Annu Rev Genomics Hum Genet 2001;2:129-51.
2. Jaeken J, Matthijs G. Congenital disorders of glycosylation: a rapidly expanding disease family. Annu Rev Genomics Hum Genet 2007;8:261-78.

3. Matthijs G, Schollen E, Bjursell C, et al. Mutations in PMM2 that cause congenital disorders of glycosylation, type Ia (CDG-Ia). Hum Mutat 2000;16:386-94.

4. Haeuptle MA, Hennet T. Congenital disorders of glycosylation: an update on defects affecting the biosynthesis of dolichol-linked oligosaccharides. Hum Mutat 2009;30:1628-41.

5. Vals MA, Pajusalu S, Kals M, et al. The Prevalence of PMM2-CDG in Estonia Based on Population Carrier Frequencies and Diagnosed Patients. JIMD Rep 2018;39:13-7.

6. Schollen E, Kjaergaard S, Legius E, et al. Lack of HardyWeinberg equilibrium for the most prevalent PMM2 mutation in CDG-Ia (congenital disorders of glycosylation type Ia). European Journal of Human Genetics 2000;8:367-71.

7. Jaeken J, van Eijk HG, van der Heul C, et al. Sialic aciddeficient serum and cerebrospinal fluid transferrin in a newly recognized genetic syndrome. Clin Chim Acta 1984;144:245-7.

8. Jaeken J, Hennet T, Matthijs G, et al. CDG nomenclature: time for a change! Biochim Biophys Acta 2009;1792:825-6.

9. Freeze HH, Chong JX, Bamshad MJ, et al. Solving glycosylation disorders: fundamental approaches reveal complicated pathways. Am J Hum Genet 2014;94:161-75.

10. Jaeken J, Péanne R. What is new in CDG? J Inherit Metab Dis 2017;40:569-86.

11. Cylwik B, Naklicki M, Chrostek L, et al. Congenital disorders of glycosylation. Part I. Defects of protein N-glycosylation. Acta Biochim Pol 2013;60:151-61.

12. Helenius A, Aebi M. Intracellular functions of $\mathrm{N}$-linked glycans. Science 2001;291:2364-9.

13. Schiff M, Roda C, Monin ML, et al. Clinical, laboratory and molecular findings and long-term follow-up data in 96 French patients with PMM2-CDG (phosphomannomutase 2-congenital disorder of glycosylation) and review of the literature. J Med Genet 2017;54:843-51.

14. Barone R, Carrozzi M, Parini R, et al. A nationwide survey of PMM2-CDG in Italy: high frequency of a mild neurological variant associated with the L32R mutation. J Neurol 2015;262:154-64.

15. Dercksen M, Crutchley AC, Honey EM, et al. ALG6CDG in South Africa: Genotype-Phenotype Description of Five Novel Patients. JIMD Rep 2013;8:17-23.

16. El-Battari A, Prorok M, Angata K, et al. Different 
glycosyltransferases are differentially processed for secretion, dimerization, and autoglycosylation. Glycobiology 2003;13:941-53.

17. Duncker IM, Asteggiano C, Freeze H. Congenital Disorders of Glycosylation. In: Mora-Montes H. Editor. Glycans: Biochemistry, Characterization and Applications Congenital Disorders of Glycosylation. Hauppauge: Nova Science, 2012;59-82.

18. Jaeken J, Rymen D, Matthijs G. Congenital disorders of glycosylation: other causes of ichthyosis. Eur J Hum Genet 2014;22:444-4.

19. Rymen D, Jaeken J. Skin manifestations in CDG. J Inherit Metab Dis 2014;37:699-708.

20. Miller BS, Khosravi MJ, Patterson MC, et al. IGF system in children with congenital disorders of glycosylation. Clin Endocrinol (Oxf) 2009;70:892-7.

21. de Lonlay P, Seta N, Barrot S, et al. A broad spectrum of clinical presentations in congenital disorders of glycosylation I: a series of 26 cases. J Med Genet 2001;38:14-9.

22. Kjaergaard S, Müller J, Skovby F. Prepubertal growth in congenital disorder of glycosylation type Ia (CDG-Ia). Arch Dis Child 2002;87:324-7.

23. Grünewald S, Imbach T, Huijben K, et al. Clinical and biochemical characteristics of congenital disorder of glycosylation type Ic, the first recognized endoplasmic reticulum defect in $\mathrm{N}$-glycan synthesis. Ann Neurol 2000;47:776-81.

24. Marquardt T, Denecke J. Congenital disorders of glycosylation: review of their molecular bases, clinical presentations and specific therapies. Eur J Pediatr 2003;162:359-79.

25. Kristiansson B, Stibler H, Hagberg B, et al. CDGS-1--a recently discovered hereditary metabolic disease. Multiple organ manifestations, incidence $1 / 80,000$, difficult to treat. Lakartidningen 1998;95:5742-8.

26. Marquardt T, Hülskamp G, Gehrmann J, et al. Severe transient myocardial ischaemia caused by hypertrophic cardiomyopathy in a patient with congenital disorder of glycosylation type Ia. Eur J Pediatr 2002;161:524-7.

27. Romano S, Bajolle F, Valayannopoulos V, et al. Conotruncal heart defects in three patients with congenital disorder of glycosylation type Ia (CDG Ia). J Med Genet 2009;46:287-8.

28. Truin G, Guillard M, Lefeber DJ, et al. Pericardial and abdominal fluid accumulation in congenital disorder of glycosylation type Ia. Mol Genet Metab 2008;94:481-4.

29. Krasnewich D, O'Brien K, Sparks S. Clinical features in adults with congenital disorders of glycosylation type Ia (CDG-Ia). Am J Med Genet 2007;145C:302-6.

30. Krasnewich D, Gahl WA. Carbohydrate-deficient glycoprotein syndrome. Adv Pediatr 1997;44:109-40.

31. Enns GM, Steiner RD, Buist N, et al. Clinical and molecular features of congenital disorder of glycosylation in patients with type 1 sialotransferrin pattern and diverse ethnic origins. J Pediatr 2002;141:695-700.

32. Pérez J de J, Udeshi ND, Shabanowitz J, et al. O-GlcNAc modification of the coat protein of the potyvirus Plum pox virus enhances viral infection. Virology 2013;442:122-31.

33. Erlandson A, Bjursell C, Stibler H, et al. Scandinavian CDG-Ia patients: genotype/phenotype correlation and geographic origin of founder mutations. Hum Genet 2001;108:359-67.

34. Monin ML, Mignot C, De Lonlay P, et al. 29 French adult patients with PMM2-congenital disorder of glycosylation: outcome of the classical pediatric phenotype and depiction of a late-onset phenotype. Orphanet J Rare Dis 2014;9:207.

35. Thompson DA, Lyons RJ, Russell-Eggitt I, et al. Retinal characteristics of the congenital disorder of glycosylation PMM2-CDG. J Inherit Metab Dis 2013;36:1039-47.

36. Kjaergaard S, Schwartz M, Skovby F. Congenital disorder of glycosylation type Ia (CDG-Ia): phenotypic spectrum of the R141H/F119L genotype. Arch Dis Child 2001;85:236-9.

37. Mohamed M, Theodore M, Claahsen-van der Grinten $\mathrm{H}$, et al. Thyroid function in PMM2-CDG: diagnostic approach and proposed management. Mol Genet Metab 2012;105:681-3.

38. Schoffer KL, O'Sullivan JD, McGill J. Congenital disorder of glycosylation type Ia presenting as early-onset cerebellar ataxia in an adult. Mov Disord 2006;21:869-72.

39. Barone R, Sturiale L, Fiumara A, et al. Borderline mental development in a congenital disorder of glycosylation (CDG) type Ia patient with multisystemic involvement (intermediate phenotype). J Inherit Metab Dis 2007;30:107-7.

40. Coman D, McGill J, MacDonald R, et al. Congenital disorder of glycosylation type 1a: three siblings with a mild neurological phenotype. J Clin Neurosci 2007;14:668-72.

41. Miller BS, Freeze HH. New disorders in carbohydrate metabolism: congenital disorders of glycosylation and their impact on the endocrine system. Rev Endocr Metab Disord 2003;4:103-13.

42. Shanti B, Silink M, Bhattacharya K, et al. Congenital disorder of glycosylation type Ia: heterogeneity in the clinical presentation from multivisceral failure to hyperinsulinaemic hypoglycaemia as leading symptoms 
in three infants with phosphomannomutase deficiency. J

Inherit Metab Dis 2009;32 Suppl 1:S241-51.

43. de Zegher F, Jaeken J. Endocrinology of the carbohydratedeficient glycoprotein syndrome type 1 from birth through adolescence. Pediatr Res 1995;37:395-401.

44. Kristiansson B, Stibler H, Wide L. Gonadal function and glycoprotein hormones in the carbohydratedeficient glycoprotein (CDG) syndrome. Acta Paediatr 1995;84:655-9.

45. de Lonlay P, Seta N. The clinical spectrum of phosphomannose isomerase deficiency, with an evaluation of mannose treatment for CDG-Ib. Biochim Biophys Acta 2009;1792:841-3.

46. Marques-da-Silva D, Francisco R, Webster D, et al. Cardiac complications of congenital disorders of glycosylation (CDG): a systematic review of the literature. J Inherit Metab Dis 2017;40:657-72.

47. de Koning TJ, Toet M, Dorland L, et al. Recurrent nonimmune hydrops fetalis associated with carbohydratedeficient glycoprotein syndrome. J Inherit Metab Dis 1998;21:681-2.

48. Jaeken J, Matthijs G, Saudubray JM, et al. Phosphomannose isomerase deficiency: a carbohydratedeficient glycoprotein syndrome with hepatic-intestinal presentation. Am J Hum Genet 1998;62:1535-9.

49. Niehues R, Hasilik M, Alton G, et al. Carbohydratedeficient glycoprotein syndrome type Ib. Phosphomannose isomerase deficiency and mannose therapy. J Clin Invest 1998;101:1414-20.

50. Babovic-Vuksanovic D, Patterson MC, Schwenk WF, et al. Severe hypoglycemia as a presenting symptom of carbohydrate-deficient glycoprotein syndrome. J Pediatr 1999;135:775-81.

51. de Lonlay P, Cuer M, Vuillaumier-Barrot S, et al. Hyperinsulinemic hypoglycemia as a presenting sign in phosphomannose isomerase deficiency: A new manifestation of carbohydrate-deficient glycoprotein syndrome treatable with mannose. J Pediatr 1999; $135: 379-83$.

52. Jaeken J, Lefeber D, Matthijs G. Clinical utility gene card for: ALG6 defective congenital disorder of glycosylation. Eur J Hum Genet 2015;23:17.

53. Williams OW, Sharafkhaneh A, Kim V, et al. Airway mucus: From production to secretion. Am J Respir Cell Mol Biol 2006;34:527-36.

54. Rafaelsen S, Johansson S, Ræder H, et al. Longterm clinical outcome and phenotypic variability in hyperphosphatemic familial tumoral calcinosis and hyperphosphatemic hyperostosis syndrome caused by a novel GALNT3 mutation; case report and review of the literature. BMC Genet 2014;15:98.

55. Huegel J, Sgariglia F, Enomoto-Iwamoto M, et al. Heparan sulfate in skeletal development, growth, and pathology: the case of hereditary multiple exostoses. Dev Dyn 2013;242:1021-32.

56. Cartault F, Munier P, Jacquemont ML, et al. Expanding the clinical spectrum of B4GALT7 deficiency: homozygous p.R270C mutation with founder effect causes Larsen of Reunion Island syndrome. Eur J Hum Genet 2015;23:49-53.

57. Farrow EG, Imel EA, White KE. Miscellaneous noninflammatory musculoskeletal conditions. Hyperphosphatemic familial tumoral calcinosis (FGF23, GALNT3 and $\alpha$ Klotho). Best Pract Res Clin Rheumatol 2011;25:735-47.

58. Ichikawa S, Sorenson AH, Austin AM, et al. Ablation of the Galnt3 gene leads to low-circulating intact fibroblast growth factor 23 (Fgf23) concentrations and hyperphosphatemia despite increased Fgf23 expression. Endocrinology 2009;150:2543-50.

59. Boccuto L, Aoki K, Flanagan-Steet H, et al. A mutation in a ganglioside biosynthetic enzyme, ST3GAL5, results in salt \& pepper syndrome, a neurocutaneous disorder with altered glycolipid and glycoprotein glycosylation. Hum Mol Genet 2014;23:418-33.

60. Harlalka GV, Lehman A, Chioza B, et al. Mutations in B4GALNT1 (GM2 synthase) underlie a new disorder of ganglioside biosynthesis. Brain 2013;136:3618-24.

61. Kato M, Saitsu H, Murakami Y, et al. PIGA mutations cause early-onset epileptic encephalopathies and distinctive features. Neurology. Neurology 2014;82:1587-96.

62. Maydan G, Noyman I, Har-Zahav A, et al. Multiple congenital anomalies-hypotonia-seizures syndrome is caused by a mutation in PIGN. J Med Genet 2011;48:383-9.

63. Almeida AM, Murakami Y, Layton DM, et al. Hypomorphic promoter mutation in PIGM causes inherited glycosylphosphatidylinositol deficiency. Nat Med 2006; 12:846-51.

64. Hansen L, Tawamie H, Murakami Y, et al. Hypomorphic mutations in PGAP2, encoding a GPI-anchor-remodeling protein, cause autosomal-recessive intellectual disability. Am J Hum Genet 2013;92:575-83.

65. Johnston JJ, Gropman AL, Sapp JC, et al. The phenotype of a germline mutation in PIGA: the gene somatically mutated in paroxysmal nocturnal hemoglobinuria. Am J Hum Genet 2012;90:295-300.

66. Krawitz PM, Murakami Y, Hecht J, et al. Mutations in 
PIGO, a member of the GPI-anchor-synthesis pathway, cause hyperphosphatasia with mental retardation. Am J Hum Genet 2012;91:146-51.

67. Kvarnung M, Nilsson D, Lindstrand A, et al. A novel intellectual disability syndrome caused by GPI anchor deficiency due to homozygous mutations in PIGT. J Med Genet 2013;50:521-8.

68. Ng BG, Hackmann K, Jones MA, et al. Mutations in the glycosylphosphatidylinositol gene PIGL cause CHIME syndrome. Am J Hum Genet 2012;90:685-8.

69. Tarailo-Graovac M, Sinclair G, Stockler-Ipsiroglu S, et al. The genotypic and phenotypic spectrum of PIGA deficiency. Orphanet J Rare Dis 2015;10:23.

70. Bessler M, Mason PJ, Hillmen P, et al. Paroxysmal nocturnal haemoglobinuria $(\mathrm{PNH})$ is caused by somatic mutations in the PIG-A gene. EMBO J 1994;13:110-7.

71. Brodsky RA. Paroxysmal nocturnal hemoglobinuria. Blood 2014;124:2804-11.

72. Sturiale L, Barone R, Garozzo D. The impact of mass spectrometry in the diagnosis of congenital disorders of glycosylation. J Inherit Metab Dis 2011;34:891-9.

73. Lefeber DJ, de Brouwer APM, Morava E, et al. Autosomal recessive dilated cardiomyopathy due to DOLK mutations results from abnormal dystroglycan O-mannosylation. PLoS Genet 2011;7:e1002427.

74. Sakharkar MK, Chow VTK, Kangueane P. Distributions of exons and introns in the human genome. In Silico Biol (Gedrukt) 2004;4:387-93.

75. Yang Y, Muzny DM, Reid JG, et al. Clinical whole-exome sequencing for the diagnosis of mendelian disorders. $\mathrm{N}$ Engl J Med 2013;369:1502-11.

76. Lionel AC, Costain G, Monfared N, et al. Improved diagnostic yield compared with targeted gene sequencing panels suggests a role for whole-genome sequencing as a first-tier genetic test. Genet Med 2018;20:435-43.

77. Dragojlovic N, Elliott AM, Adam S, et al. The cost and diagnostic yield of exome sequencing for children with suspected genetic disorders: a benchmarking study. Genet Med 2018;20:1013-21.

78. Kalia SS, Adelman K, Bale SJ, et al. Recommendations for reporting of secondary findings in clinical exome and genome sequencing, 2016 update (ACMG SF v2.0): a policy statement of the American College of Medical Genetics and Genomics. Genet Med 2017;19:249-55.

79. Rothstein MA. Currents in contemporary ethics. GINA, the ADA, and genetic discrimination in employment. J Law Med Ethics 2008;36:837-40.

80. Tamminga RY, Lefeber DJ, Kamps WA, et al. Recurrent thrombo-embolism in a child with a congenital disorder of glycosylation (CDG) type Ib and treatment with mannose. Pediatr Hematol Oncol 2008;25:762-8.

81. Mention K, Lacaille F, Valayannopoulos V, et al. Development of liver disease despite mannose treatment in two patients with CDG-Ib. Mol Genet Metab 2008;93:40-3.

82. Sharma V, Nayak J, DeRossi C, et al. Mannose supplements induce embryonic lethality and blindness in phosphomannose isomerase hypomorphic mice. FASEB J 2014;28:1854-69.

83. Schroeder AS, Kappler M, Bonfert M, et al. Seizures and stupor during intravenous mannose therapy in a patient with CDG syndrome type $1 \mathrm{~b}$ (MPI-CDG). J Inherit Metab Dis 2010;33 Suppl 3:S497-502.

84. Etzioni A, Tonetti M. Fucose supplementation in leukocyte adhesion deficiency type II. Blood 2000;95:3641-3.

85. Almeida A, Layton M, Karadimitris A. Inherited glycosylphosphatidyl inositol deficiency: a treatable CDG. Biochim Biophys Acta 2009;1792:874-80.

86. Wong SY, Gadomski T, van Scherpenzeel M, et al. Oral D-galactose supplementation in PGM1-CDG. Genet Med 2017;19:1226-35.

87. Morelle W, Potelle S, Witters P, et al. Galactose Supplementation in Patients With TMEM165-CDG Rescues the Glycosylation Defects. J Clin Endocrinol Metab 2017;102:1375-86.

88. Park JH, Hogrebe M, Fobker M, et al. SLC39A8 deficiency: biochemical correction and major clinical improvement by manganese therapy. Genet Med 2018;20:259-68.

89. Niethamer TK, Yardeni T, Leoyklang P, et al. Oral monosaccharide therapies to reverse renal and muscle hyposialylation in a mouse model of GNE myopathy. Mol Genet Metab 2012;107:748-55.

90. Brasil S, Pascoal C, Francisco R, et al. CDG Therapies: From Bench to Bedside. Int J Mol Sci 2018;19:1304.

91. Krasnewich D. Human glycosylation disorders. Marino PA, editor. Cancer Biomark 2014;14:3-16.

92. Almeida AM, Murakami Y, Baker A, et al. Targeted therapy for inherited GPI deficiency. N Engl J Med 2007;356:1641-7.

93. Joshi C, Kolbe DL, Mansilla MA, et al. Ketogenic diet - A novel treatment for early epileptic encephalopathy due to PIGA deficiency. Brain Dev 2016;38:848-51.

Cite this article as: Chang IJ, He M, Lam CT. Congenital disorders of glycosylation. Ann Transl Med 2018;6(24):477. doi: 10.21037/atm.2018.10.45 